\title{
Preventing functional loss during immobilization after osteoporotic wrist fractures in elderly patients: a randomized clinical trial
}

Nadja Schott ${ }^{*}$ and Heide Korbus

\begin{abstract}
Background: Distal radius fractures are among the most common fractures and account for approximately one-sixth of all fractures diagnosed. Therapy results after distal radius fracture, especially of elderly patients, are often suboptimal. The inevitable immobilization for several weeks leads to reduction in range of motion, deterioration of muscle strength, malfunction of fine motor skills as well as changes of motor and sensory representations in the brain. Currently, there are no strategies to counteract these immobilization problems. The overall aim of the study is to investigate the therapeutic potential of motor-cognitive approaches (mental practice or mirror therapy) on hand function after wrist fracture.

Methods/Design: This study is a controlled, randomized, longitudinal intervention study with three intervention groups. One experimental group imagines movements of the fractured upper extremity without executing them (mental practice). The second experimental group receives a mirror therapy program consisting of the performance of functional movement synergies using the unaffected forearm, wrist, and hand. The control group completes a relaxation training regime. Additionally, all patients receive usual care by the general practitioner. We include women aged 60 years and older having a distal radius fracture and sufficient cognitive function. All groups are visited at home for therapy sessions 5 times per week for the first 3 weeks and 3 times per week for weeks 4 to 6 . Measurements are taken at therapy onset, and after 3,6 and 12 weeks. The primary outcome measure will assess upper extremity functioning (Patient-Rated Wrist Evaluation [PRWE]), while secondary outcome measures cover subjective wrist function (Disabilities of the Arm and Shoulder; [DASH], objective impairment (range of motion, grip force) and quality of life (EuroQol-5D, [EQ5D]).
\end{abstract}

Discussion: Results from this trial will contribute to the evidence on motor-cognitive approaches in the early therapy of distal radius fractures.

Trial registration: The trial is registered at ClinicalTrials.gov with registration number NCT01394809 and was granted permission by the Medical Ethical Review Committee of the University of Tübingen in June 2011.

Keywords: Distal radius fracture, Mental practice, Mirror therapy, Immobilization, Women $\geq 60$ years, Clinical trial

\footnotetext{
* Correspondence: nadja.schott@inspo.uni-stuttgart.de

Department of Sports and Exercise Science, University of Stuttgart,

Allmandring 28, 70569 Stuttgart, Germany
}

(c) 2014 Schott and Korbus; licensee BioMed Central Ltd. This is an Open Access article distributed under the terms of the Creative Commons Attribution License (http://creativecommons.org/licenses/by/4.0), which permits unrestricted use, distribution, and reproduction in any medium, provided the original work is properly credited. The Creative Commons Public Domain Dedication waiver (http://creativecommons.org/publicdomain/zero/1.0/) applies to the data made available in this article, unless otherwise stated. 


\section{Background}

Distal radius fractures are among the most common osteoporotic fractures [1] and account for an estimated $17 \%$ of fractures treated in US emergency departments [2] with a female-male ratio of about 3:1 [3]. Corresponding to the demographic development, osteoporotic fractures of the wrist, humerus spine or hip can be expected to increase further in the coming years and with it the burden on healthcare resources [4,5]. Regardless of the fact whether these fractures are treated surgically or by casting, patients are at least immobilized for two to six weeks or more. Physical and occupational therapy as a key element in rehabilitation starts after the removal of the fixation device. However, during the period of fixation, patients often keep their injured hand in rigid postures, in which the volar plates and adjacent ligaments of the digital joints are shortened [6]. Different methods of treatment, but especially the long immobilization periods lead to overall complication rates ranging from 6 to $80 \%$ and have been associated with poor functional outcomes [7]. These complications not only include complex and regional pain syndrome, stiffness, nerve injury, tendon and ligament injuries, but a massive reduction in range of motion (ROM), muscular atrophy, and loss of movement representation [8]. As a result, the final hand function is often suboptimal [9]. Previous studies have indicated that $20 \%$ of patients with distal radius fracture had persistent symptoms, and 10\% continued to have functional impairments after the typical recovery period [10]. In a study by consortium partners we demonstrated that the risk of losing independence after a wrist fracture is almost as high as after a hip fracture [11]. This partly relates to upper extremity dysfunction with activities of daily living such as eating, getting dressed and washed.

The goal for rehabilitation after wrist fractures is to achieve complete and rapid recovery of ROM, strength, and function of the wrist and hand. Improvement of the functional outcome after wrist fracture can probably not only be found in changing the operative technique [12]. Hence, for improvement of functional outcome, one has to focus on the postoperative rehabilitation period $[13,14]$. A patient would need a treatment procedure that is more active without actually stressing the bone and that may prevent from the negative side effects as well as from the central reorganization that takes place as a result of immobilization. This leads to a temporary forgetting of the function of the affected limb [15], and results in the inefficiency of the central control of movements. Immobilization has shown to result rather rapidly in changes of motor and sensory representations in the brain of peripheral organs such as finger, arm, or leg $[16,17]$. For example, Langer et al. [16] showed a decrease in cortical thickness in the left primary motor and somatosensory area as well as a decrease in the grey matter in the left corticospinal tract after at least 14 days of limb immobilization.

Several studies have shown that sensory input does not exclusively result from actually performed movements. Imagined movements without actually moving the limbs (explicit motor imagery) as well as observational learning (mirror therapy) also generate sensory input [18-21]. Mirror therapy (MT), in which a mirror is placed in the patient's midsagittal plane, so that he/she can see his/her unaffected arm/hand as if it was the affected one, has mainly been studied for two different purposes: pain relief [22], and motor recovery poststroke [23-25]. Furthermore, MT has been shown to increase ipsilateral primary motor excitability in healthy controls [26], which may account for the improvement in motor function. Mental practice (MP) represents a class of training or therapy regimes in which an internal representation of a movement is repeatedly simulated in mind from a first-person perspective, without actual physical movement, and is effective in motor recovery in neurological and orthopedic rehabilitation [27-29]. Proposed mechanisms for improved motor recovery with MT and MP include reconciliation of motor output and sensory feedback MT, [30] and graded activation of cortical motor networks MP, [31]. According to Jeannerod $[32,33] \mathrm{MP}$ and the preparation of movements share common mechanisms and are functionally equivalent [34]. Furthermore, the activation of pre-motor "mirror neurons", which have intimate connections with visual processing areas, is thought to prime the primary motor cortex and to be important in imitating motor action [35-37].

In orthopedic rehabilitation MP as well as MT has only received minor attention as a promising psychological complement to conventional physical therapy approaches. Three studies have examined the effects of MT in patients after hand surgery other than amputation [18,38,39]. While Rosén and Lundborg [38] reported three different cases (e.g. tendon repair) when MT was applied in combination with traditional hand training (no further details regarding the number of training sessions or duration were given), Altschuler and $\mathrm{Hu}$ [18] as well as Rostami et al. [39] examined hand function with different orthopedic conditions in one (insufficient information on intervention), respectively 12 patients ( 15 sessions à 30 minutes). Both studies conducted MT in combination with physical therapy. All three studies reported improvements in objective as well as subjective measures of hand function. None of these studies had the goal to overcome the effects of immobilization. There are mixed results on the effects of MP during disuse or immobilization [40-45]. Some researchers have found support for mental imagery to maintain muscle strength and flexibility $[42,44$ Schott \& 
Limberger: Effects of mental practice on gait after total hip endoprosthesis, in prep], while others have not $[40,41,43,45]$. Methodological inconsistencies could account for many of the contradictory findings within the literature. Studies vary in the participants' age and health status, the type of imagery, the content of imagery, as well as intervention length. Explicit imagery has been shown to create the greatest physiological benefits but only few studies specifically elaborate on their imagery script. Protocols for imagery intervention also varied among researchers from as little as 10 days to 7 weeks in duration with varying numbers of sessions per day. To our knowledge, only two studies examined the efficacy of explicit motor imagery and MT so far $[42,46]$. Ietswaart and colleagues [46] found no enhanced improvement as a result of MP with motor imagery in upper hand function of stroke patients. Frenkel [42] examined the efficacy of a MP combined with MT after knee endoprothesis surgery, but only found significant results for the criterion flexion. No other studies exist with regard to patients with orthopedic injuries, comparing the efficacy of MT or MP.

The purpose of this randomized study is to determine whether explicit motor imagery or MT during the immobilization period after distal radius fracture results in a greater recovery of central aspects of hand function. The objective of this study is to establish the effectiveness of daily training of movement imagery with the affected arm (MP) or the healthy arm (MT). It will also be examined whether enhanced functional recovery of the hand due to MP or MT is also associated with increases in the amount of activities of daily living. It will be investigated whether the benefit of motor-cognitive approaches for distal radius fracture patients is related to their individual differences in motor imagery ability.

\section{Methods/Design}

Post-fracture strategies that tackle the problems mentioned above might reduce the functional loss after wrist fractures and improve quality of life considerably. Currently, no proactive strategies are published to counteract the immobilization problems. Our participants are being recruited from different hospitals and randomly allocated to one of three groups. There are two experimental intervention groups (MP and MT) and one control group. We use two different strategies originally developed and tested for the rehabilitation of stroke patients: Participants either receive a MP or a MT program. This novel approach combines neuropsychological approaches with sport science and conventional physiotherapy.

The study is conducted as a randomized controlled trial. The purpose of the study is to examine the effects of MP and MT on upper extremity function after osteoporotic wrist fractures.
The trial is conducted in agreement with the principles of the Declaration of Helsinki, and with the guidelines of Good Clinical Practice (GCP). The study protocol was approved by the local and independent Ethics Committee Tübingen (Institutional Review Board).

\section{Procedure}

All patients give written informed consent prior to the study. Before randomization, the patients complete the Controllability of Motor Imagery Test [47] to control for the ability to perform and control a mental image of a movement, which is essential for successful imagery training. After baseline assessment, randomization is performed by an independent department (Department of Biometrics at Ulm University).

Assessment of primary and secondary outcomes takes place upon entry to the study (T1) by a blinded assessor and is repeated after 3 weeks (T2), 6 weeks (T3), and 12 weeks (T4, follow-up) after the beginning of the training program (see Figure 1).

\section{Participants}

We include females aged 60 years and older with a distal radius fracture. We exclude persons with the following characteristics:

- Unstable medical conditions which preclude surgical intervention (ASA 5)

- Patients with an open fracture

- Associated soft tissue or skeletal injury to the same limb

- Cognitive impairment 6CIT [48],

\section{Intervention}

Participants in the experimental groups receive either a MP or a MT program. Patients in the control group are instructed to practice relaxation techniques. The intervention period lasts six weeks and takes place in the patient's private home. Additionally, all patients receive therapy as usual. All MP and MT training sessions were provided by the same physical therapist; however, a second therapist conducted the primary and secondary evaluations. In week one to three the patient participates in five sessions per week on five consecutive days (Monday-Friday), and in week's four to six the patient participates three times per week with the therapist and two times without. As of week 4 , when the guided therapy sessions are reduced to 3 times per week, all patients receive written instructions containing descriptions and photos of the movement tasks. They are asked to document their training sessions (duration and level of difficulty) in daily training diaries. Additionally, the patients are supplied with an MP3-player with a text for progressive muscle relaxation. All therapy sessions are 45 minutes per treatment session. Each 


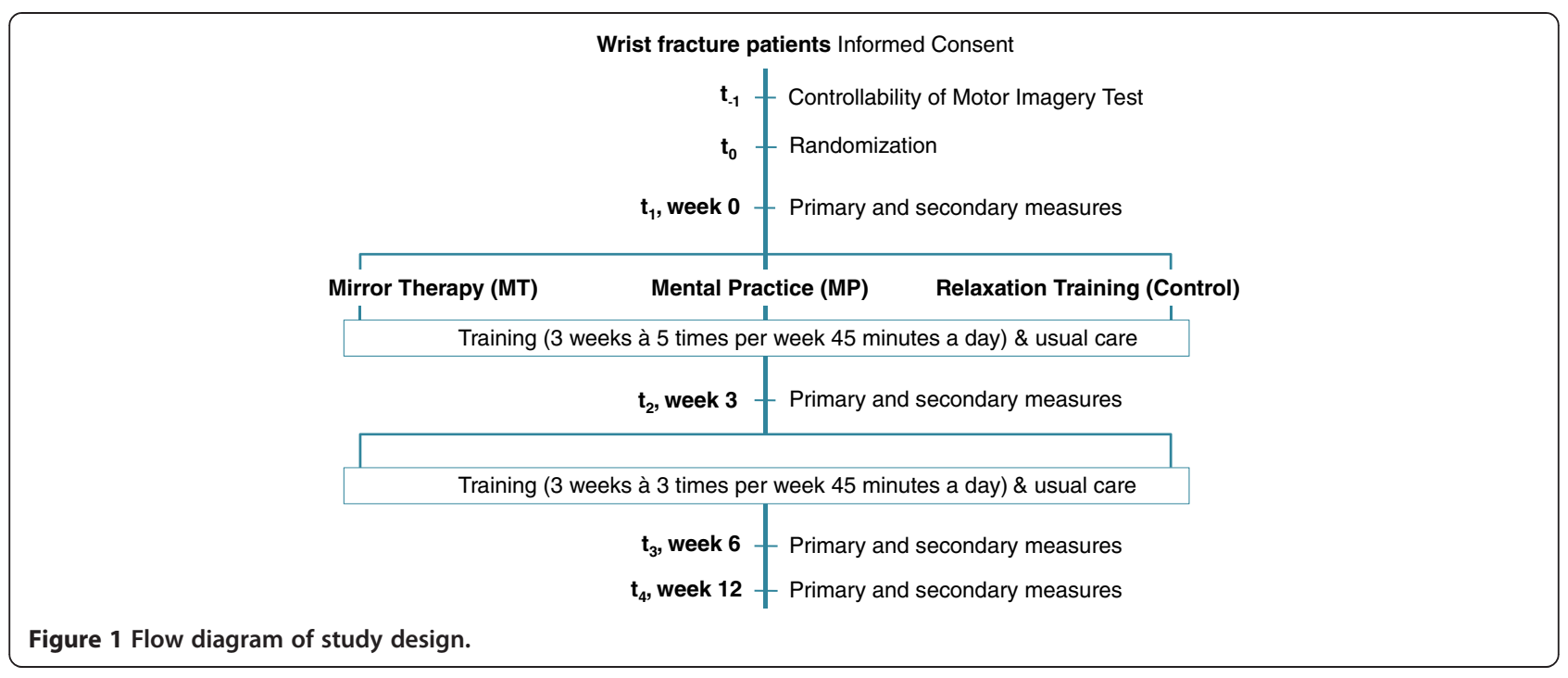

session (MP as well as MT) starts with a standardized progressive muscle relaxation focusing on wrist (healthy side) and other body parts (based on [49]), and is applied for five to ten minutes to aid the participant's ability to concentrate on the internal body sensations of the following imagination protocol.

The content of the two motor-cognitive interventions (MP and MT) is based on a simplified version of Gentile's task taxonomy [50]: four sections are defined by the combination of stationary/mobile performer with open/closed environment. The basic movement tasks in this study are dorsal extension, palmar flexion, radial and ulnar abduction as well as supination and pronation using Gentile's Taxonomy of Tasks to structure the training content and progression ([51]; details provided in Table 1). Object manipulation and/or intertrial variability mark higher levels of difficulty. Patients are asked to rate the level of difficulty for mentally simulating the movement, respectively for perceiving the reflected image in the mirror as their affected side (1 very easy to 10 very hard). As soon as they rate a task with 4 ("reasonably easy") or lower, they try a task of a higher difficulty level. For retaining same conditions, the patients should not execute the movement with the affected side during MP and MT.

The training protocol for MT and MP after distal radius fracture follows the standardized method of the Mental Gait Training [43,52]. It consists of several sequential steps: (1) description and (2) instruction of wrist movements; (3) the physical training with the unaffected side in alteration with either MP (with both wrists) or MT (only with the unaffected side); (4) building and strengthening of the motor representation of wrist movements, and (5) the physical execution of wrist movements (providing that the actual sensation does not disturb the mental image) (see Figure 2).
In a first step the therapist discusses with the patient wrist movements the patient executes on a typical day. Both develop a therapy plan together in order to define which movements should be addressed during therapy. In the next step the patient is confronted with the physiological model of wrist movements, the movement descriptions. A movement description is the objective, biomechanical representation of the wrist movement using here the unaffected hand of the patient as a model. This method is used to provide the relevant functional aspects of the reference movement. Additionally, in order to achieve greater learning and retention results, the patients are prompted to verbalize the movements from an individual inside view addressing as many sensory modalities from memory as possible. The next step consists of the elaboration of the key characteristics of the different wrist movements. Finally, the key characteristics are marked symbolically, i.e. they are renamed as individual short formulae (e.g. up - centered or pull release for dorsal flexion). The aim is the compression of the information (chunking). The image should be approximated thus to the dynamism and the temporal duration of the real movement [54].

After finalizing the movement descriptions the patients in the MP group execute movements with their unaffected side to provide additional feedback such as posture, muscle tension, or joint positions. The physical training distinguishes itself by a sort of proprioceptive training in which under modification of perception, movement and environment the movement representation should be further stabilized and differentiated. The key characteristics for flexion were up-centered, and for the ulnar and radial deviation inwards-centered-outwards-centered. Following the physical training with open and closed eyes to enhance kinesthetic perception, the patients in the MP group imagined normal wrist 


\begin{tabular}{|c|c|c|}
\hline & \multicolumn{2}{|c|}{ Body stability } \\
\hline & No object manipulation & Object manipulation \\
\hline & $\Rightarrow$ dorsal extension & $\Rightarrow$ lifting up weight (dorsal extension) \\
\hline & $\Rightarrow$ palmar flexion & $\Rightarrow$ lifting up weight (palmar flexion) \\
\hline & $\Rightarrow$ ulnar-/radial abduction & $\Rightarrow$ wiping a table with a cloth \\
\hline & $\Rightarrow$ supination/pronation & $\Rightarrow$ handling of a bike lock/kitchen timer \\
\hline No intertrial variability & $\begin{array}{l}\Rightarrow \text { opening and closing hand } \\
\text { (like grasping a cup) }\end{array}$ & $\Rightarrow$ wringing out a towel \\
\hline & $\Rightarrow$ thumb-finger-oppositions & $\Rightarrow$ compressing a softball \\
\hline & $\Rightarrow$ finger-tapping sequence & $\begin{array}{l}\Rightarrow \text { grasping a pen with little finger (e.g.) } \\
\text { and thumb }\end{array}$ \\
\hline & & $\Rightarrow$ playing piano (simple 5-finger-scale) \\
\hline \multicolumn{3}{|l|}{ Stationary environment } \\
\hline & $\begin{array}{l}\Rightarrow \text { Dorsal extension against different } \\
\text { resistances }\end{array}$ & $\begin{array}{l}\Rightarrow \text { grasping, lifting, turning different cups } \\
\text { (size, weight) }\end{array}$ \\
\hline & $\begin{array}{l}\Rightarrow \text { Palmar flexion against different } \\
\text { resistances }\end{array}$ & $\begin{array}{l}\Rightarrow \text { catching different balls on a marple run } \\
\text { (reaction) }\end{array}$ \\
\hline & $\begin{array}{l}\Rightarrow \text { Ulnar-/radial abduction against } \\
\text { different resistances }\end{array}$ & $\begin{array}{l}\Rightarrow \text { compressing softballs of different } \\
\text { sizes/hardness }\end{array}$ \\
\hline Intertrial variability & $\begin{array}{l}\Rightarrow \text { Supination/pronation against } \\
\text { different resistances }\end{array}$ & $\begin{array}{l}\Rightarrow \text { pulling a cloth across the table (ulnar/radial) } \\
\text { against different resistances }\end{array}$ \\
\hline & $\Rightarrow$ Swimming in turbulent water & $\Rightarrow$ bowling/rolling a ball towards a partner \\
\hline & & $\Rightarrow$ playing ping-pong \\
\hline & & $\Rightarrow$ juggling with one hand \\
\hline
\end{tabular}

movement with the unaffected side with different imagery modalities (kinesthetic, visual; for examples see Table 2) with the following sequence (modified from [43]):

1. Active execution with the unaffected side ( $1 \mathrm{x}$ with open eyes)

2. Visual imagery (1 x unaffected side, $1 \times$ affected side; with closed eyes)

3. Active execution with the unaffected side (1 x with closed eyes)

4. Kinesthetic imagery (1 x unaffected side, $1 \mathrm{x}$ affected side; with closed eyes).

After establishing the internal concept of wrist movements, the systematic training phase with physical training alternating with MP follows this protocol:

1. Active execution with the unaffected side ( $2 \mathrm{x}$ with open eyes)

2. Active execution with the unaffected side ( $2 \times$ with closed eyes)

3. Kinesthetic imagery with the unaffected side ( $1 \mathrm{x}$ with closed eyes)

4. Kinesthetic imagery with the affected side (5-10 x with closed eyes)
Due to the findings of Yae et al. [55], that mental imagery is most effective with a kinesthetic focus, our training process is aiming at that modality.

Patients in the MT group immediately start with the observation of the movements with the unaffected side in the mirror. During MT the affected hand remains hidden from view behind the mirror, which is placed in the midsagittal plane of the participant. The patient is observing the reflected image of the unaffected hand, which appears like the affected side performing the movement. Patients perform each wrist movement with the unaffected side 8 to 10 times with visual focus on the mirrored picture of the healthy side.

The overall aim of these protocols is to preserve motor representation of hand function to enable patients to execute wrist movements with high accuracy and appropriate strength after splint removal.

The control group receives relaxation training to achieve the same total amount of time the therapist spends with the patients of the experimental groups.

\section{Outcome measures}

The assessments focus on changes in three key domains: subjective wrist function (PRWE-G; DASH), objective 


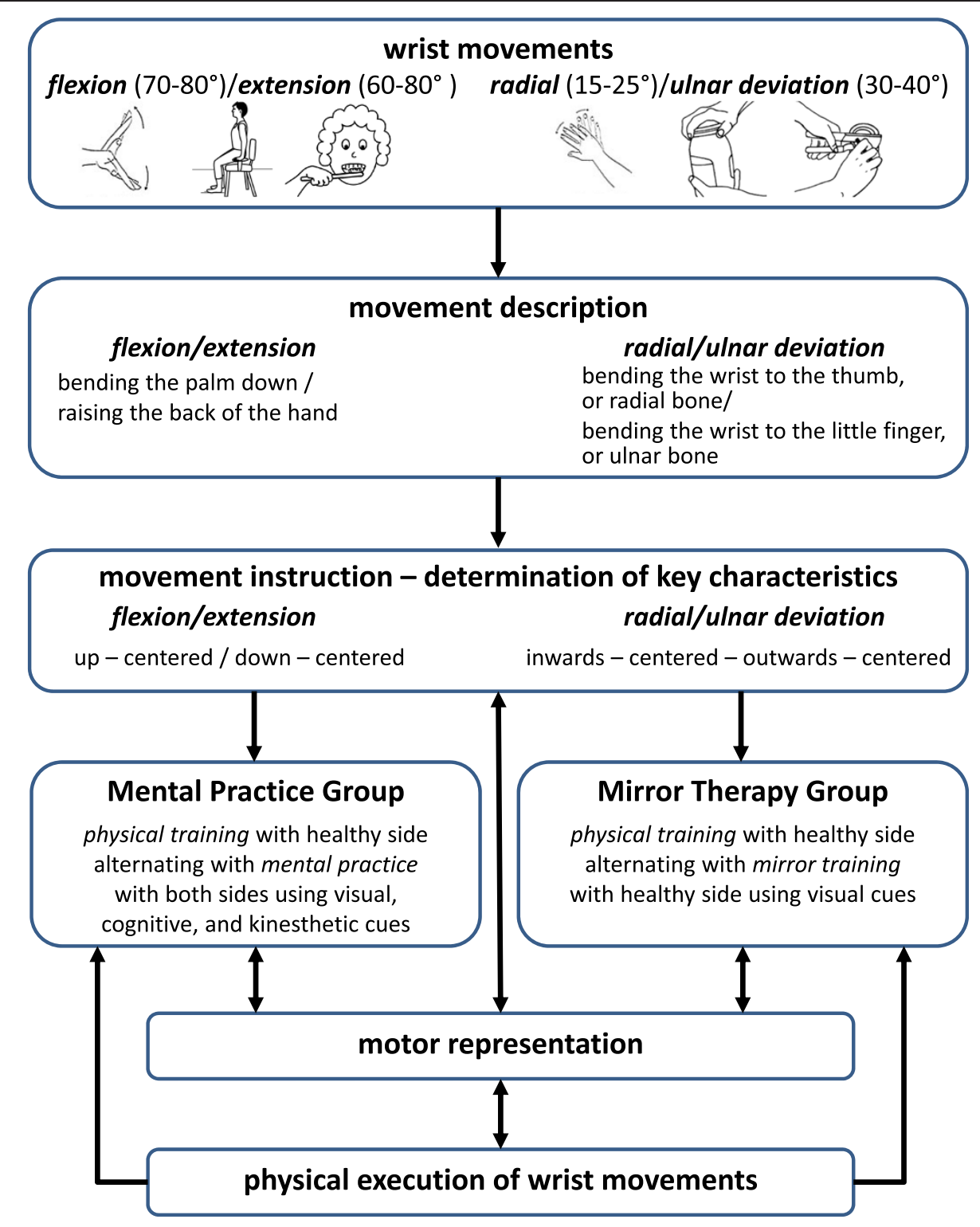

Figure 2 MT and MP procedure after distal radius fracture (modified from $[43,52,53]$ ).

impairment (Range of motion, grip strength) and quality of life/social engagement (DASH, EuroQol).

\section{Primary outcome}

Is the subjective rating of pain and impairment in activities of daily life (Patient-rated Wrist Evaluation; PRWE-G, German version, [56]), a validated tool for assessing functional outcome in patients with distal radius fracture [57]. The questionnaire is completed by the investigator via patient interview and consists of two domains, pain and function. There are five items in the pain domain (e.g. "Rate your pain when lifting a heavy object.") and ten items in the function domain (e.g. "Rate your difficulty you experience when fastening buttons on your shirt.").
The response to each item is scored on a scale of 0 to 10 . Scores of each individual item were provided with qualitative descriptors defined as: none (0), minimal (1-2), mild (3-4), moderate (5-6), severe (7-8) or very severe (9-10). The pain score is the sum of five items, with the worst possible score of 50, and the disability (function) score is the sum of ten items divided by 2 .

\section{Secondary outcomes}

The Disabilities of the Arm, Shoulder and Hand (DASH; [58]) score is a 11-item, self-administered questionnaire designed to measure physical functions, symptoms, and social function, work, sleep, and confidence items in patients with any or several musculoskeletal disorders of 


\begin{tabular}{|c|c|}
\hline Visual: & $\begin{array}{l}\text { How does your hand look turning a door key? } \\
\text { Imagine seeing your hand, the key, and the door. } \\
\text { See your wrist turning and the forearm pronating. }\end{array}$ \\
\hline Kinesthetic: & $\begin{array}{l}\text { Imagine touching the cold metal of the key with your } \\
\text { fingers. Now feel your muscles in your forearm and } \\
\text { hand activate as you grasp and turn the key. } \\
\text { What can you feel? Is there a tension in your muscles? }\end{array}$ \\
\hline
\end{tabular}

the upper limb. The DASH outcome measure is scored in two components: the Disability/Symptom and the optional high performance Sport/Music module. The DASH Disability/Symptom score is a summation of the responses to 11 questions on a scale of 1 (without difficulty or no symptom) to 5 (unable to engage in activity or very severe symptom). This value is then transformed to a score out of 100 by subtracting one and multiplying by 25 with values between 0 (no disability) and 100 (severe disability). The questions examine the degree of difficulty in performing a variety of physical activities because of arm, shoulder, or hand problems (6 items). It also investigates the severity of pain, tingling ( 2 items), as well as the effect of the upper limb problem on social activities, work, and sleep (3 items).

Range of motion (ROM) of the radiocarpal joint is measured on both sides with a handheld goniometer. Four parameters are evaluated: dorsal extension, palmar flexion, radial and ulnar abduction of the wrist joint [59].

Grip strength is measured with a handheld dynamometer (Myon, Prophysics), and carried out three trials on each side. If necessary, the instrument is adjusted to the size of the patients' hands. The patient is sitting in a chair, her upper arm by her side of the body and the forearm stretched to an angle of $90^{\circ}$, with the elbow supported. The patient is encouraged to squeeze the dynamometer as hard as possible. A mean value is determined according to the standardized Mathiowetz procedure [60]. The grip strength is presented as the percentage of the value of the injured side of the value of the uninjured side. In order to account for hand dominance in grip strength, if the nondominant hand was injured, the percentage will be multiplied by 1.10; if the dominant hand was injured, the percentage will be multiplied by 0.90 [61].

Social engagement is evaluated with the social functioning subscale of the DASH-Questionnaire [58] and health related quality of life with the EQ-5D [62]. The EQ-5D is a short questionnaire with five dimensions (mobility, self-care, usual activities, pain or discomfort, and anxiety or depression), each of which can be rated at one of three levels ( 1 no problem, 2 some problems, 3 extreme/severe problems). EQ-5D utility scores range between a full health score of 1 (where the respondent has no problems on any dimension) and the lowest score of -0.59 (when the respondent reports that they are at the bottom level of each dimension).

Additionally, falls associated self-efficacy is evaluated (ABC-Scale, German version, [63]). Demographic information is analyzed at the beginning of the intervention period.

\section{Sample size/power calculations}

The sample size calculation was approximated with a $3 \times 3$-factorial ANOVA-approach based on the primary outcome. Previous randomised controlled trials of therapy interventions have shown effect sizes $\geq .80$ in similar populations for the PRWE as a result of hand therapy, however therapy starting weeks after the accident. To prove an intervention effect with about medium effect size of Cohen's $\mathrm{f}=0.25$ with an error probability $\alpha=0.05$, a power $\beta=0.8$, a correlation between repeated measures of .05 , and a nonsphericity correction at $1, \mathrm{n} \approx 12$ people in each study arm are required for analysis. The analysis was carried out with the G*Power software (G*Power V 3.1.6 Franz Faul, Universität Kiel, Kiel, Germany). To account for potential attrition, we will inflate the sample size by $10 \%$, resulting in a total sample size of 39 with 13 participants allocated to each group.

\section{Statistical analysis}

Data will be analyzed using (M)ANOVA with repeated measures to ascertain the effects of therapy on outcome variables and to follow improvements individually in time. An Intention-to-treat principle will be applied. Missing data will be replaced by an imputation method for missing measurements.

\section{Discussion}

This trial is due to deliver results by the end of 2014. Up to now, participants have shown a very high compliance, less than 10\% resigned (1 in MP group, 1 in CG). Recruitment is more difficult than expected, partly caused by a very short hospital stay combined with the dependency of prompt communication of new adequate patients, partly due to an exceptionally mild winter (less falls), and partly due to communication deficits. Therefore, the intervention period turns out to be several months longer than initially planned.

\section{Conclusions}

Study outcomes should be carefully analyzed, possibly leading to further adjustments of the intervention protocol or to a second trial that is ruling out the assumed reasons for inconclusive results like a high rate of intercurrent diseases. A positive study outcome should be tested in a large multi-center trial. 


\section{Competing interests}

The authors declare that they have no competing interests.

\section{Authors' contributions}

NS is grant holder; additional intellectual contributions were made by HK. Both authors are site investigators who contributed to the development and writing of the protocol to its final version. Both authors have been involved in the drafting and revision of this manuscript and have given approval of the final manuscript.

The authors thank PD Dr. Clemens Becker for his support in the conception of this study.

\section{Acknowledgements and funding}

This study is supported by the German Federal Ministry of Education and Research [01EC1007D]. The views expressed in this paper are those of the authors and may not be shared by the funding bodies. The study is part of the project "Prevention and Rehabilitation of Osteoporotic Fractures in disadvantaged Populations" (PROFinD).

We owe special thanks to C. Becker, B. Kinner and U. Liener, who provide access to appropriate wrist facture patients, to the team of the Department of Biometrics at UIm University for randomization, to A. Brechenser for performing of the relaxation training in the control group, and T. Klotzbier for performing the assessments.

\section{Received: 5 August 2014 Accepted: 26 August 2014}

Published: 30 August 2014

\section{References}

1. Cummings SR, Kelsey JL, Nevitt MC, O'Dowd KJ: Epidemiology of osteoporosis and osteoporotic fractures. Epidemiol Rev 1985, 7:178-208.

2. Chung KC, Spilson SV: The frequency and epidemiology of hand and forearm fractures in the United States. J Hand Surg Am 2001, 26(5):908-915.

3. Brogren E, Petranek M, Atroshi I: Incidence and characteristics of distal radius fractures in a southern Swedish region. BMC Musculoskelet Disord 2007, 8:48

4. Morin SN, Lix LM, Majumdar SR, Leslie WD: Temporal trends in the incidence of osteoporotic fractures. Curr Osteoporos Rep 2013, 11(4):263-269.

5. Shauver MJ, Yin H, Banerjee M, Chung KC: Current and future national costs to medicare for the treatment of distal radius fracture in the elderly. J Hand Surg Am 2011, 36(8):1282-1287.

6. Freeland $A E$, Luber $K T$ : Biomechanics and biology of plate fixation of distal radius fractures. Hand Clin 2005, 21(3):329-339.

7. McKay SD, MacDermid JC, Roth JH, Richards RS: Assessment of complications of distal radius fractures and development of complication checklist. J Hand Surg Am 2001, 26:916-922.

8. Diaz-Garcia RJ, Oda T, Shauver MJ, Chung KC: A systematic review of outcomes and complications of treating unstable distal radius fractures in the elderly. J Hand Surg Am 2011, 36(5):824-835.

9. Meine J: Die Früh- und Spätkomplikationen der Radiusfraktur loco classico. Z Unfallchir Versicherungsmed Berufskr 1989, 82:25-32.

10. Kuo LC, Yang TH, Hsu YY, Wu PT, Lin CL, Hsu HY, Jou IM: Is progressive early digit mobilization intervention beneficial for patients with external fixation of distal radius fracture? A pilot randomized controlled trial. Clin Rehabil 2013, 27(11):983-993.

11. Einsiedel T, Becker C, Stengel D, Schmelz A, Kramer M, Däxle M, Lechner F, Kinzl L, Gebhard F: Frakturen der oberen Extremität beim geriatrischen Patienten - Harmlose Monoverletzung oder Ende der Selbstständigkeit? Z Gerontol Geriat 2006, 39:451-461.

12. Schneppendahl J, Windolf J, Kaufmann RA: Distal radius fractures: current concepts. J Hand Surg Am 2012, 37:1718-1725.

13. Dekkers $M$, Soballe K: Activities and impairments in the early stage of rehabilitation after Colles' fracture. Disabil Rehabil 2004, 26:662-668.

14. Smith DW, Brou KE, Henry MH: Early active rehabilitation for operatively stabilized distal radius fractures. J Hand Ther 2004, 17(1):43-49.

15. de Jong BM, Coert JH, Stenekes MW, Leenders KL, Paans AM, Nicolai JP: Cerebral reorganisation of human hand movement following dynamic immobilisation. Neuroreport 2003, 14:1693-1696.

16. Langer N, Hänggi J, Müller NA, Simmen HP, Jäncke L: Effects of limb immobilization on brain plasticity. Neurology 2012, 78(3):182-188.
17. Ngomo S, Leonard G, Mercier C: Influence of the amount of use on hand motor cortex representation: Effects of immobilization and motor training. Neurosci 2012, 220:208-214.

18. Altschuler EL, Hu J: Mirror therapy in a patient with a fractured wrist and no active wrist extension. Scand J Plast Reconstr Surg Hand Surg 2008, 42:110-111.

19. Barclay-Goddard RE, Stevenson TJ, Poluha W, Thalman L: Mental practice for treating upper extremity deficits in individuals with hemiparesis after stroke. Stroke 2011, 42(11):e574-e575.

20. Fritzsch C, Wang J, Dos Santos LF, Mauritz KH, Brunetti M, Dohle C: Different effects of the mirror illusion on motor and somatosensory processing. Restor Neurol Neurosci 2014, 32(2):269-280.

21. Porro CA, Francescato MP, Cettolo V, Diamond ME, Baraldi P, Zuiani C, Bazzocchi M, di Prampero PE: Primary motor and sensory cortex activation during motor performance and motor imagery: a functional magnetic resonance imaging study. J Neurosc 1996, 16(23):7688-7698.

22. Moseley GL: Graded motor imagery for pathologic pain. A randomized controlled trial. Neurology 2006, 67(12):2129-2134.

23. Dohle C, Pullen J, Nakaten A, Kust J, Rietz C, Karbe H: Mirror therapy promotes recovery from severe hemiparesis: a randomized controlled trial. Neurorehabil Neural Repair 2009, 23:209-217.

24. Ezendam D, Bongers RM, Jannink MJ: Systematic review of the effectiveness of mirror therapy in upper extremity function. Disabil Rehabil 2009, 31(26):2135-2149.

25. Rothgangel AS, Braun SM, Beurskens AJ, Seitz RJ, Wade DT: The clinical aspects of mirror therapy in rehabilitation: a systematic review of the literature. Int J Rehabil Res 2011, 34(1):1-13.

26. Garry MI, Loftus A, Summers JJ: Mirror, mirror on the wall: viewing a mirror reflection of unilateral hand movements facilitates ipsilateral M1 excitability. Exp Brain Res 2005, 163:118-122.

27. Braun S, Kleynen M, Van Heel T, Kruithof N, Wade D, Beurskens A: The effects of mental practice in neurological rehabilitation; a systematic review and meta-analysis. Front Hum Neurosci 2013, 7:390.

28. Malouin F, Jackson PL, Richards CL: Towards the integration of mental practice in rehabilitation programs. A critical review. Front Hum Neurosci 2013, 7:576.

29. Mayer J, Bohn J, Görlich P, Eberspächer H: Mental gait training - effectiveness of a therapy method in the rehabilitation after hip-replacement. Zeitschrift für Orthopädie 2005, 143:419-423.

30. McCabe CS, Haigh RC, Ring EFR, Halligan PW, Wall PD, Blake DR: A controlled pilot study of the utility of mirror visual feedback in the treatment of Complex Regional Pain Syndrome (Type 1). Rheumatology 2003, 42:97-101.

31. Liepert J, Bauder H, Wolfgang HR, Miltner WH, Taub E, Weiller C: Treatmentinduced cortical reorganization after stroke in humans. Stroke 2000, 31:1210-1216.

32. Jeannerod M: The representing brain: neural correlates of motor intention and imagery. Brain Behav Sci 1994, 17:187-245.

33. Jeannerod M: Mental imagery in the motor context. Neuropsychologia 1995, 33:1419-1433.

34. Vogt S, Di Rienzo F, Collet C, Collins A, Guillot A: Multiple roles of motor imagery during action observation. Front Hum Neurosci 2013, 7(807):1-13.

35. Carvalho D, Teixeira S, Lucas M, Yuan TF, Chaves F, Peressutti C, Machado S, Bittencourt J, Menéndez-González M, Nardi AE, Velasques B, Cagy M, Piedade R, Ribeiro P, Arias-Carrión O: The mirror neuron system in poststroke rehabilitation. Int Arch Med 2013, 6(1):41.

36. Rizzolatti G, Fadiga L, Gallese V, Fogassi L: Premotor cortex and the recognition of motor actions. Cogn Brain Res 1996, 3(2):131-141.

37. Keysers C, Wicker B, Gazzola V, Anton JL, Fogassi L, Gallese V: A touching sight: SII/PV activation during the observation and experience of touch. Neuron 2004, 42:335-346.

38. Rosén B, Lundborg G: Training with a mirror in rehabilitation of the hand. Scand J Plast Reconstr Surg Hand Surg 2005, 39:104-108.

39. Rostami HR, Arefi A, Tabatabaei S: Effect of mirror therapy on hand function in patients with hand orthopaedic injuries: a randomized controlled trial. Disabil Rehabil 2013, 35(19):1647-1651.

40. Clark BC, Manini TM, Bolanowski SJ, Ploutz-Snyder LL: Adaptations in human neuromuscular function following prolonged unweighting: II. Neurological properties and motor imagery efficacy. J Appl Physiol 2006, 101:264-272. 
41. Crews RT, Kamen G: Motor-evoked potentials following imagery and limb disuse. Int J Neurosci 2006, 116:639-651.

42. Frenkel MO: Mentales Training in der orthopädischen Rehabilitation nach Knieendoprothetik. PhD Thesis. University of Heidelberg: Institute for Sport and Exercise Science; 2010

43. Frenkel MO, Herzig DS, Gebhard F, Mayer J, Becker C, Einsiedel T: Mental practice maintains range of motion despite forearm immobilization: a pilot study in healthy persons. J Rehabil Med 2014, 46(3):225-232.

44. Newsom J, Knight P, Balnave R: Use of mental imagery to limit strength loss after immobilization. J Sport Rehabil 2003, 12:249-258

45. Stenekes MW, Geertzen JH, Nicolai JP, de Jong BM, Mulder T: Effects of motor imagery on hand function during immobilization after flexor tendon repair. Arch Phys Med Rehabil 2009, 90(4):553-559.

46. letswaart M, Johnston M, Dijkerman HC, Joice S, Scott CL, MacWalter RS, Hamilton SJ: Mental practice with motor imagery in stroke recovery: randomized controlled trial of efficacy. Brain 2011, 134:1373-1386.

47. Schott N: German test of the controllability of motor imagery (GT-CMI) in older adults. Z Gerontol Geriatr 2013, 46(7):663-672.

48. Brooke $\mathrm{P}$, Bullock R: Validation of a 6 item cognitive impairment test with a view to primary care usage. Int I Geriatr Psychiatry 1999, 14:936-940.

49. Jacobson E: Entspannung als Therapie: Progressive Relaxation in Theorie und Praxis. München: Pfeiffer; 1990.

50. Gentile AM: Skill acquisition: Action, movement, and neuromotor processes. In Movement science: Foundations for Physical Therapy. 2nd edition. Edited by Carr JH, Shepherd RB. Rockville, MD: Aspen; 2000:111-187.

51. Schott N, Frenkel MO, Korbus H, Francis K: Mental Practice in Orthopaedic Rehabilitation: Where, What, and How? A case report. Science et Motricité 2013, 82:93-103.

52. Mayer J, Görlich P, Eberspächer H: Mentales Gehtraining. Berlin: Springer; 2003.

53. Eberspächer H: Mentales Training. 8th edition. München: Copress Sport; 2012.

54. Mayer J, Hermann HD: Mentales Training. Heidelberg: Springer; 2011.

55. Yao WX, Ranganathan VK, Allexandre D, Siemionow V, Yue GH: Kinesthetic imagery training of forceful muscle contractions increases brain signal and muscle strength. Front Hum Neurosci 2013, 7:561.

56. John M, Angst F, Awiszus F, Pap G, MacDermid JC, Simmen BR: The patient-rated wrist evaluation (PRWE): cross-cultural adaptation into German and evaluation of its psychometric properties. Clin Exp Rheumatol 2008, 26(6):1047-1105.

57. Changulani M, Okonkwo U, Keswani T, Kalairajah Y: Outcome evaluation measures for wrist and hand: which one to choose? Int Orthop 2008, 32(1):1-6.

58. Germann G, Harth A, Wind G, Demir E: Standardisation and validation of the German version 2.0 of the Disability of Arm, Shoulder, Hand (DASH) questionnaire. Unfallchirurg 2003, 106:13-19.

59. Gajdosik R, Bohannon R: Clinical measurement of range of motion: review of goniometry emphasising reliability and validity. Phys Ther 1987, 67:1867-1872.

60. Mathiowetz V, Weber K, Volland G, Kashman N: Reliability and validity of grip and pinch strength evaluations. J Hand Surg Am 1984, 9:222-226.

61. Fike ML, Rousseau E: Measurement of adult hand strength: a comparison of two instruments. Occup Ther J Res 1982, 2:43-49.

62. The EuroQol Group: EuroQol - a new facility for the measurement of health-related quality of life. Health Policy 1990, 16(3):199-208.

63. Schott N: Reliability and validity of the German short version of the Activities specific Balance Confidence (ABC) scale in older adults. Arch Gerontol Geriatr 2014. ePub 4 June.

\section{Submit your next manuscript to BioMed Central and take full advantage of:}

- Convenient online submission

- Thorough peer review

- No space constraints or color figure charges

- Immediate publication on acceptance

- Inclusion in PubMed, CAS, Scopus and Google Scholar

- Research which is freely available for redistribution

Submit your manuscript at www.biomedcentral.com/submit
C Biomed Central 